\title{
MusculoskeletalDisordersandTreatment
}

RESEARCH ARTICLE

\section{Differences in Myofascial Trigger Points and Algometry in Craniocervical Muscles among Children with and without Headache: A Comparative Study}

\author{
Raquel Vega-Plá ${ }^{1 *}$, Aitziber Rubio-Aramendi ${ }^{2}$, Silvia Cardiel-Sanchéz ${ }^{2,3}$, Isabel Albarova-Corral ${ }^{2,4}$, \\ Sara Cabanillas-Barea ${ }^{5}$ and Miguel Malo-Urriés ${ }^{6}$
}

\author{
${ }^{1}$ Centro de Fisioterapia María Moreno, Calatayud, Zaragoza, Spain \\ ${ }^{2}$ In-Forma Fisioterapia, San Mateo de Gállego, Zaragoza, Spain \\ ${ }^{3}$ Professor, Universidad San Jorge, Villanueva de Gállego, Zaragoza, Spain \\ ${ }^{4}$ Professor, Universidad Pública de Navarra, Tudela, Navarra, Spain \\ ${ }^{5}$ Professor, Departamento de Fisioterapia, Universidad Internacional de Catalunya, Barcelona, Spain \\ ${ }^{6}$ Professor, Departamento de Fisiatría y Enfermería, Universidad de Zaragoza, Zaragoza, Spain
}

*Corresponding author: Raquel Vega-Plá, Centro de Fisioterapia María Moreno, Calatayud 50300, Zaragoza, Spain

\begin{abstract}
Background: Headache is the most frequent neurological symptoms in children. The relationship between craniocervical muscles and headache has been studied in adults, however, studies in children are limited.

Objectives: The present study aimed to examine and compare the presence of active and latent myofascial triggers points in head, neck, and shoulder muscles and pain pressure thresholds in children with headache and healthy controls.

Methods: A comparative study with non experimental design was conducted. A total of 126 participants between 3 and 16-years-old were recruited. Subjects were divided into two groups: Children with headaches (who had suffered it in the last three months) and children without a headache. Anthropometric data, presence and number of latent or active myofascial trigger points, and pressure pain threshold in craniocervical muscles were compared between both groups.

Results: A total of 19 symptomatic and 107 asymptomatic children were studied. The present study evidenced significant differences in the symptomatic group for the presence of both active and latent myofascial trigger points in the upper trapezius, sternocleidomastoid, suboccipital, and splenium muscles. No significant differences in the
\end{abstract}

presence of myofascial trigger points in masseter and temporalis muscles were found. No statistically significant differences were found in algometry data between both groups.

Conclusion: Despite the fact that pressure pain threshold did not present any differences between both groups, children with headache presented a greater number of active or latent myofascial trigger points in craniocervical muscles.

\section{Keywords}

Children, Headache, Myofascial trigger points, Pressure pain threshold

\begin{abstract}
Abbreviations
ICHD-3: International Classification of Headache Disorders; MTP: Myofascial Trigger Point; PPT: Pressure Pain Threshold; MDC: Minimum Detectable Change
\end{abstract}

\section{Introduction}

Headache is one of the most frequent neurological symptoms and a common manifestation of pain in childhood, with a high risk of developing into a chronic condition during adulthood [1]. In the third edition of the International Classification of Headache Disorders

Citation: Vega-Plá R, Rubio-Aramendi A, Cardiel-Sánchez S, Albarova-Corral I, Cabanillas-Barea S, et al. (2021) Differences in Myofascial Trigger Points and Algometry in Craniocervical Muscles among Children with and without Headache: A Comparative Study. J Musculoskelet Disord Treat 7:103. doi. org/10.23937/2572-3243.1510103

Accepted: August 11, 2021: Published: August 13, 2021

Copyright: (c) 2021 Vega-Plá, et al. This is an open-access article distributed under the terms of the Creative Commons Attribution License, which permits unrestricted use, distribution, and reproduction in any medium, provided the original author and source are credited. 
(ICHD-3), headache was divided into primary disorders, without an underlying cause, such as migraine or tension-type headache, and secondary disorders, attributable to a specific aetiology $[2,3]$.

Although headache is rare before the age of 4 , its prevalence increases throughout childhood, reaching a peak at about 13 years of age in both sexes [4]. Between $6.5 \%$ and $30 \%$ of children and adolescents report headaches at least once a week [1]. Prevalence during childhood and adolescence may vary according to classification systems and methods of diagnosis. However, many studies agree that, migraine and tensiontype headache are the most frequent conditions [5]. Between $3 \%$ and $11 \%$ of children suffer migraine, whilst between $10 \%$ and $24 \%$ suffer tension-type headache [1]. A high percentage of children who have headache at ages between 7 and 15 years will continue to suffer from migraine attacks years later [4].

Headaches, especially migraines, have a significant socioeconomic and personal impact, related in the case of children to the loss of school days, school performance, and ability to participate in social or extracurricular activities $[2,6]$. This generates a decrease in the children's quality of life compared to their healthy peers [1].

Headaches are usually related to other physical or psychological symptoms including dysfunctional family situations, poor physical condition, physical or emotional abuse, bullying, problematic relationships at school, lack of free time, and in the case of adolescents, excessive alcohol and tobacco consumption. The literature has also shown the relevance of craniocervical muscles, cervical mobility, and posture in children with headaches $[7,8]$.

Different studies have evidenced the importance of the referred pain elicited by Myofascial Trigger Points (MTPs) of temporalis, suboccipital, upper trapezius, or sternocleidomastoid muscles in adults with headache. Referred pain from active MTPs can provoke the headache. It is evidenced that hyperexcitability of peripheral and central nociceptive pain pathways plays an essential role in headaches $[9,10]$.

Different studies have also related headache and Pressure Pain Threshold (PPT) in pericranial and extracephalic regions [11-13]. PPT reflects sensitivity and can be objectively measured by pressure algometry using an algometer. Pressure algometry has been shown to be a valid and reliable measurement of PPT in craniocervical muscles [14].

Despite extensive research of MTPs and PPT in adults with headache, studies on MTPs and PPT in children are limited. Previous studies showed that children with headache presented a higher number of active MTPs in upper trapezius, sternocleidomastoid, and temporalis muscles, although their activity was not related to pain intensity $[9,15,16]$. These studies did not find significant differences between the right and left sides, although it was observed that muscle referred pain areas were broader in children than in adults $[9,15,16]$. There is limited evidence that indicates the influence of active MTPs in children's headache, however, the samples were small, and did not study the relationship between the presence of MTPs and their PPT. Therefore, our purpose was to examine and compare the presence of active and latent MTPs in head, neck, and shoulder muscles and pain pressure threshold in children with headache and healthy controls.

\section{Methods}

A cross-sectional observational study was designed to assess the presence of myofascial trigger points and the pressure pain threshold in the craniocervical region in children with headache compared to asymptomatic controls. The study was conducted in accordance with the Declaration of Helsinki and approved by the local ethics committee (Comité Ético de Investigación Clínica de Aragón) (PI19/048).

\section{Subjects}

Children were recruited from "Bajo Gállego" School after consultation. Written informed consent was provided by their parents. Potential participants were informed of their right to refuse to participate in the study or to withdraw consent to participate at any time without reprisal.

A total of 126 participants between 3 and 16-yearsold were recruited. All children or adolescents who accepted to participate were included in the study, as no more inclusion or exclusion criteria were described.

A brief clinical history was registered in an interview with both children and parents, mainly to know if the children had suffered headaches in the last three months so that they were divided into two groups: Children who had suffered headaches in the last three months (children with headache) and children who had no suffered headaches in the last three months (children without headaches). Children with headache filled out a standardised headache questionnaire to know the characteristics of their headache (intensity, frequency, duration). Furthermore, the age and a series of anthropometric data (sex, weight, height) were collected.

\section{Variables}

Six craniocervical muscles were evaluated bilaterally: Upper trapezius, suboccipital, capitis splenius, sternocleidomastoid, masseter, and temporalis. MTPs were evaluated following the criteria described by Simons, et al. (1999): 1) Presence of a palpable taut band in a skeletal muscle; 2) Presence of a hyperirritable tender spot within the taut band; 3) Local twitch response elicited by the snapping palpation of the taut band; and 
Table 1: Demographic and clinical data of the sample.

\begin{tabular}{|c|c|c|c|}
\hline Variables & Symptomatic group $(n=19)$ & Asymptomatic group $(n=107)$ & $p$ Value \\
\hline $\operatorname{Sex}(n)$ & & & 0.110 \\
\hline Male & 13 & 65 & \\
\hline Female & 6 & 61 & \\
\hline Age (years), mean \pm SD & $11.8 \pm 2.6$ & $11.3 \pm 2.85$ & 0.394 \\
\hline Height $(\mathrm{cm})$, mean \pm SD & $154.9 \pm 14.8$ & $147.5 \pm 16.5$ & $0.032^{*}$ \\
\hline Weight $(\mathrm{Kg})$, mean \pm SD & $52.8 \pm 15.3$ & $44.4 \pm 15$ & $0.007^{\star}$ \\
\hline
\end{tabular}

Note: *Level of significance, $p<0.05$; SD: Standard Deviation.

4) Presence of referred pain in response to trigger point compression [17]. Trigger points were considered active if both the local and the referred pain evoked by the compression reproduced the spontaneous symptoms of the children and the elicited pain was recognised by the children. Trigger points were considered latent if the local and referred pain elicited by the compression did not reproduce any pain symptom familiar to the children [17].

Cervical PPT was measured using an analogical algometer with a round surface area of $1 \mathrm{~cm}$ [2], with pressure applied at a rate of $1 \mathrm{~kg} / \mathrm{cm}^{2} / \mathrm{s}$ perpendicular to the skin. Participants were instructed to raise their hand at the precise moment that pressure sensation changed to pain. A 30-second resting period was allowed between each measure. The mean of 3 trials was calculated over each point and used for analysis [11]. The reliability of PPT measurement is high (intraclass correlation coefficient $=0.91-0.97$ ) [18] and minimum detectable change (MDC) for PPT over the cervical spine in patients with neck pain is $47.2 \mathrm{kPa}$ [12] PPT is a precise and reliable (Cronbach's alpha $\geq 0.92$ ) measurement in children [19].

In order to optimise reliability, the same researcher with orthopaedic manual therapy specialised training and more than 10 years of experience, performed the outcome measures. This investigator was blinded to the allocation group of each participant.

\section{Data analysis}

Data were analysed using SPSS 25.0 statistics software. Variables were analysed using descriptive statistics (mean, standard deviation, medians, percentages, and total numbers). A comparative study was performed. The Kolmogorov-Smirnov test was used to determine the normality of data distribution. To analyse quantitative variables, an unpaired T-test was used for normally distributed data, and the MannWhitney $U$ test used when this was not the case. Chisquare test was used to analyse qualitative variables. Data collection was processed and analyzed with a level of confidence of $95 \%$, setting $p$-value at the 0.05 level.

\section{Results}

A total of 126 participants between 3 and 16-years- old were recruited and agreed to participate (19 symptomatic children and 107 asymptomatic children). No child was excluded from the study. The characteristics of the sample are shown in Table 1. Interestingly, the headache group presented significantly higher height and weight than the control group ( $p=0.007)$.

Subjects with headaches showed a greater number of MTPs than controls $(p<0.001)$. The mean number of myofascial trigger points (MTPs) in the symptomatic group was $3.95 \pm 3.77$, while in the asymptomatic group was $1.16 \pm 2.14$ (Table 2). Within the symptomatic group, MTPs in the right sternocleidomastoid (active $21.1 \%$; latent $26.3 \%$ ), right (active $42.1 \%$; latent $36.8 \%$ ) and left (active 15.8\%; latent 26.3\%) upper trapezius, right splenium (active 10.5\%; latent $25.8 \%$ ) and right suboccipital muscles (active 10.5\%; latent $26.3 \%$ ) were the most prevalent. Within the asymptomatic group, the most prevalent MTPs were located in the right sternocleidomastoid (active 1.9\%; latent 11.2\%) and in the right upper trapezius (active $4.7 \%$; latent $15 \%$ ) (Table 2). Differences in the presence of MTPs were significant for right sternocleidomastoid $(p<0.001)$, right $(p<0.001)$ and left $(p<0.001)$ upper trapezius, right $(p=0.011)$ and left $(p=0.048)$ splenius and right suboccipital $(p=0.003)$. The symptomatic group showed a greater percentage of active and latent MTPs than the control group. No significant differences in the presence of active or latent MTPs in masseter and temporalis muscles were found (Table 2).

No statistically significant differences were found in algometry data for sternocleidomastoid, masseter, temporalis, upper trapezius, splenium, or suboccipital muscles (Table 3).

\section{Discussion}

The current cross-sectional observational study showed the existence of a higher number of active and latent MTPs in the craniocervical region of children with headaches. Height, weight, and number of MTPs in sternocleidomastoid, upper trapezius, splenius and suboccipital muscles were significantly greater in the symptomatic group. No significant differences in the presence of MTPs were found in masseter and temporalis muscles nor algometry data.

In our sample, children with headache presented 
Table 2: Descriptive data (total numbers and percentages) and comparative analysis ( $p$-Value) for myofascial trigger points (active or latent) among symptomatic and asymptomatic group.

\begin{tabular}{|c|c|c|c|c|c|}
\hline \multicolumn{3}{|c|}{ Myofascial Trigger Points n (\%) } & $\begin{array}{l}\text { Symptomatic group } \\
(n=19)\end{array}$ & $\begin{array}{l}\text { Asymptomatic group } \\
(\mathrm{n}=107)\end{array}$ & $p$ Value \\
\hline \multirow{6}{*}{ Sternocleidomastoid } & \multirow{3}{*}{ Right } & Active & $4(21.1)$ & $2(1.9)$ & \multirow{3}{*}{$<0.001$} \\
\hline & & Latent & $5(26.3)$ & $12(11.2)$ & \\
\hline & & No MTP & $10(52.6)$ & 93 (96.9) & \\
\hline & \multirow{3}{*}{ Left } & Active & $2(10.5)$ & 0 & \multirow{3}{*}{$0.094^{*}$} \\
\hline & & Latent & $1(5.3)$ & $6(5.6)$ & \\
\hline & & No MTP & $16(84.5)$ & $101(94.4)$ & \\
\hline \multirow{6}{*}{ Masseter } & \multirow{3}{*}{ Right } & Active & 0 & $4(3.7)$ & \multirow{3}{*}{0.419} \\
\hline & & Latent & $1(5.3)$ & $8(7.5)$ & \\
\hline & & No MTP & $18(94.7)$ & $95(88.8)$ & \\
\hline & \multirow{3}{*}{ Left } & Active & $1(5.3)$ & $5(4.7)$ & \multirow{3}{*}{0.940} \\
\hline & & Latent & $1(5.3)$ & $7(6.5)$ & \\
\hline & & No MTP & $17(89.5)$ & $95(88.8)$ & \\
\hline \multirow{6}{*}{ Temporalis } & \multirow{3}{*}{ Right } & Active & 0 & 0 & \multirow{3}{*}{0.394} \\
\hline & & Latent & 0 & $4(3.7)$ & \\
\hline & & No MTP & $19(100)$ & $103(96.3)$ & \\
\hline & \multirow{3}{*}{ Left } & Active & 0 & 0 & \multirow{3}{*}{0.673} \\
\hline & & Latent & 0 & $1(0.9)$ & \\
\hline & & No MTP & $19(100)$ & $106(99.1)$ & \\
\hline \multirow{6}{*}{ Trapezius } & \multirow{3}{*}{ Right } & Active & 8 (42.1) & $5(4.7)$ & \multirow{3}{*}{$<0.001$} \\
\hline & & Latent & $7(36.8)$ & $16(15)$ & \\
\hline & & No MTP & $4(21.1)$ & $86(80.4)$ & \\
\hline & \multirow{3}{*}{ Left } & Active & $3(15.8)$ & $2(1.9)$ & \multirow{3}{*}{$<0.001$} \\
\hline & & Latent & $5(26.3)$ & $8(7.5)$ & \\
\hline & & No MTP & $11(57.9)$ & $97(90.7)$ & \\
\hline \multirow{6}{*}{ Splenium } & \multirow{3}{*}{ Right } & Active & $2(10.5)$ & $1(0.9)$ & \multirow{3}{*}{$0.011^{*}$} \\
\hline & & Latent & $3(25.8)$ & $7(6.5)$ & \\
\hline & & No MTP & $14(73.7)$ & $99(92.5)$ & \\
\hline & \multirow{3}{*}{ Left } & Active & 0 & 0 & \multirow{3}{*}{$0.048^{*}$} \\
\hline & & Latent & $2(10.5)$ & $2(1.9)$ & \\
\hline & & No MTP & $17(89.5)$ & $105(98.1)$ & \\
\hline \multirow{6}{*}{ Suboccipital } & & Active & $2(10.5)$ & 0 & \\
\hline & Right & Latent & $5(26.3)$ & $12(11.2)$ & $0.003^{*}$ \\
\hline & & No MTP & $12(63.2)$ & 95 (88.8) & \\
\hline & & Active & 0 & 0 & \\
\hline & Left & Latent & $1(2.3)$ & $3(2.8)$ & 0.575 \\
\hline & & No MTP & $18(94.7)$ & $104(97.2)$ & \\
\hline Total score & & & $3.95 \pm 3.77$ & $1.16 \pm 2.14$ & $<0.001^{\circ}$ \\
\hline
\end{tabular}

Note: *Level of significance, $p<0.05$.

Table 3: Comparison of algometry outcomes in craniocervical muscles among symptomatic and asymptomatic group.

\begin{tabular}{|l|l|l|l|l|}
\hline \multicolumn{2}{|c|}{} & $\begin{array}{l}\text { Symptomatic group } \\
\text { Algometry (KPa) }\end{array}$ & $\begin{array}{l}\text { Asymptomatic group } \\
(\mathbf{n}=\mathbf{1 9})\end{array}$ & $\boldsymbol{p}$ Value \\
\hline Sternocleidomastoid & Right & 0.92 & 0.9 & 0.892 \\
\cline { 2 - 5 } & Left & 0.94 & 0.92 & 0.933 \\
\hline Masseter & Right & 1.6 & 1.6 & 0.985 \\
\cline { 2 - 5 } & Left & 1.82 & 1.71 & 0.393 \\
\hline
\end{tabular}




\begin{tabular}{|l|l|l|l|l|}
\hline Temporalis & Right & 2.5 & 2.56 & 0.785 \\
\cline { 2 - 5 } & Left & 2.62 & 2.63 & 0.639 \\
\hline Trapezius & Right & 2.98 & 2.68 & 0.475 \\
\cline { 2 - 5 } & Left & 3.11 & 2.61 & 0.433 \\
\hline Splenium & Right & 2.21 & 2.09 & 0.951 \\
\hline & Left & 2.4 & 2.09 & 0.819 \\
\hline Suboccipital & Right & 2.35 & 2.09 & 0.892 \\
\hline & Left & 2.24 & 2.03 & 0.965 \\
\hline
\end{tabular}

a statistically significant higher mean value for height and weight compared to the asymptomatic group. Previous studies in children's populations showed a relationship between obesity and headache during childhood, just like for adult populations. Although the mechanisms underlying the relationship between obesity and headache are not yet well-understood, several physiological hypotheses have been proposed, including the role of inflammatory mediators such as interleukins and calcitonin gene-related peptide, among others [20,21]. Given the evidence of a relationship between obesity and headache, weight can be considered as a modifiable risk factor for chronic headaches. Hershey, et al. [20] and Ravid, et al. [21] found that a reduction in BMI produced a reduction in headache frequency, but not in headacherelated disability $[20,21]$. So, although obesity may be an important and modifiable factor in the outcomes of headache, further research is required.

The present study found statistically significant differences in the overall score of MTPs between both groups. Subjects with headache presented a greater number of MTPs than asymptomatic children. Previous studies such as Fernández-de-las-Peñas, et al. [16] or Alonso-Blanco, et al. [9] showed similar results to ours $[9,16]$. The present study evidenced significant differences in the symptomatic group for the presence of both active and latent MTPs in the upper trapezius, sternocleidomastoid, suboccipital, and splenium muscles. Fernández-de-las-Peñas, et al. [16] and AlonsoBlanco, et al. [9] also obtained significant differences in the masseter and temporalis muscles $[9,16]$. These authors evidenced that temporal and suboccipital muscles presented the highest prevalence of active and latent MTPs in the headache group $[9,16]$. The present study did not find significant differences in the symptomatic group for the presence of MTPs in temporal and masseter muscles. This finding could be explained through the following hypothesis, temporal and masseter muscles are innervated by trigeminal nerve, while upper trapezius, sternocleidomastoid, suboccipital, and splenium muscles are innervated by cervical nerves. It is possible that our sample have a greater sensitization in the cervical nerves, but not in the trigeminal nerve, so this could explain the greater presence of MTPs in upper trapezius, sternocleidomastoid, suboccipital, and splenium muscles and the absence of MTPs in temporal and masseter muscles. Nevertheless, further research about this topic is needed.

Different studies have shown the relevance of MTPs craniocervical muscles (suboccipital, upper trapezius, temporal, or sternocleidomastoid) related to the appearance of headaches in adults [22]. Our study in children supports that a similar relationship may exist in children and adolescents. The relationship between the presence of MTPs and the appearance of headaches has a neurophysiological basis. MTPs release inflammatory mediators, such as prostaglandins, which stimulate and sensitise nociceptive nerve endings, causing the referred pain that is perceived as headache. The sensitisation of muscle nociceptors is believed to be the primary peripheral mechanism leading to headache. Under some circumstances, the peripheral nociceptive inputs may be more prolonged and/or intense, turning into continuous afferent stimulation. In predisposed individuals, this may lead to sensitisation of the central nervous system, which may, in turn, contribute to the persistence, amplification, and spread of pain and the conversion of headache from episodic to chronic [10]. Due to the apparent involvement of MTPs in the pathogenesis of headaches, physical therapy techniques directed to inactivate MTPs, would produce a reduction in intensity, duration, and frequency of headaches, as well as prevention of perpetuation in adulthood [23].

Despite the hypothesised mechanisms, no statistically significant differences were found in PPTs in the craniocervical muscles between symptomatic and asymptomatic groups. Different studies have demonstrated that PPT is a reliable measurement of sensitivity in adults (intraclass correlation coefficient = 0.91-0.97) [18] and children (Cronbach's alpha $\geq 0.92$ ) [19]. Soee, et al. [11] and Ferracini, et al. [13] examined PPTs in pericranial and extracephalics regions in children with headache, obtaining similar results to ours. Children with headaches did not present a reduction in pressure pain threshold in the craniocervical region $[11,13]$. It has been suggested that muscle sensitivity may be greater in subjects with different types of headaches such as migraine or tension-type headache [24]. In adults with headaches, pressure pain thresholds are lower in the shoulder, neck, and pericranial regions in comparison with asymptomatic individuals. Some studies have proposed that children with headaches could present greater pain sensitivity in muscles of the shoulder and 
neck region than asymptomatic children. Nevertheless, there is limited evidence and no consensus concerning this topic $[11,13]$.

The present study did not detect any differences in PPTs between symptomatic and asymptomatic groups. This finding could be explained through different reasons. Although algometry is an objective test, each subject has a different capacity to withstand the pain regardless of its symptomatology, which can itself be influenced by different physical and behavioural, and maturative factors. Furthermore, children are less capable of defining the pain pressure threshold, as this is an aspect that will be consolidated during maturation. Besides, because of the relatively short history of headaches in children compared to adults, the children may not be sensitised.

Some potential limitations of this study should be recognised. First, headaches have not been classified and differentiated into subgroups. It would be interesting to investigate the presence of referred pain elicited by active MTPs in children with frequent episodic tension-type headache or migraine. However, headache characteristics develop over the years and classification becomes easier in adulthood. Second, a cause-and-effect relationship between MTPs and headaches cannot be established, as the design of the study was not longitudinal. Future controlled clinical studies should analyse the effects of MTPs treatment in headache to further elucidate the etiologic role of active MTPs in this population.

\section{Conclusion}

The current study showed a greater presence of active and latent MTPs in upper trapezius, sternocleidomastoid, suboccipital, and splenium muscles in children with headache compared to asymptomatic subjects. The pressure pain thresholds did not present any differences between both groups.

\section{Acknowledgments}

This research did not receive any specific grant from funding agencies in the public, commercial, or notfor-profit sectors. Authors have no financial or other relationships that might lead to a conflict of interest.

\section{Declarations of Interest}

None.

\section{References}

1. Kernick D, Reinhold D, Campbell JL (2009) Impact of headache on young people in a school population. $\mathrm{Br} \mathrm{J}$ Gen Pract 59: 678-681.

2. Headache Classification Committee of the International Headache Society (IHS) (2018) The International Classification of Headache Disorders. ( $3^{\text {rd }}$ edn $)$, Cephalalgia 38: 1-211.

3. Robbins MS, Lipton RB (2010) The epidemiology of primary headache disorders. Semin Neurol 30: 107-119.
4. Fearon P, Hotopf M (2001) Relation between headache in childhood and physical and psychiatric symptoms in adulthood: National birth cohort study. BMJ 322: 1145.

5. Hershey AD (2012) Pediatric headache: Update on recent research. Headache 52: 327-332.

6. Rousseau-Salvador C, Amouroux R, Annequin D, Salvador A, Tourniaire B, et al. (2014) Anxiety, depression and school absenteeism in youth with chronic or episodic headache. Pain Res Manag 19: 235-240.

7. Straube A, Heinen F, Ebinger F, Von Kries R (2013) Headache in school children: Prevalence and risk factors. Dtsch Arztebl Int 110: 811-818.

8. Russo A, Bruno A, Trojsi F, Tessitore A, Tedeschi G (2016) Lifestyle factors and migraine in childhood. Curr Pain Headache Rep 20: 9.

9. Alonso-Blanco $C$, Fernández-de-las-Peñas $C$, FernándezMayoralas DM, de-la-Llave-Rincón IA, Pareja JA, et al. (2011) Prevalence and anatomical localization of muscle referred pain from active trigger points in head and neck musculature in adults and children with chronic tensiontype headache. Pain Med 12: 1453-1463.

10. Arendt-Nielsen L (2015) Headache: Muscle tension, trigger points and referred pain. Int J Clin Pract 8-12.

11. Soee A-BL, Skov L, Kreiner S, Tornøe B, Thomsen LL (2013) Pain sensitivity and pericranial tenderness in children with tension-type headache: A controlled study. J Pain Res 6: 425-434.

12. Walton DM, Macdermid JC, Nielson W, Teasell RW, Chiasson M, et al. (2011) Reliability, standard error, and minimum detectable change of clinical pressure pain threshold testing in people with and without acute neck pain. J Orthop Sport Phys Ther 41: 644-650.

13. Ferracini GN, Stuginsk-Barbosa J, Dach F, Speciali JG (2014) A comparison pressure pain threshold in pericranial and extracephalic regions in children with migraine. Pain Med 15: 702-709.

14. Castien RF, Van der Wouden JC, De Hertogh W (2018) Pressure pain thresholds over thecranio-cervical region in headache: A systematic review and meta-analysis. J Headache Pain 19: 9.

15. Fernández-De-Las-Peñas $C$, Cuadrado ML, Pareja JA (2007) Myofascial trigger points, neck mobility, and forward head posture in episodic tension-type headache. Headache 47: $662-672$

16. Fernández-De-Las-Peñas $C$, Fernández-Mayoralas DM, Ortega-Santiago R, Ambite-Quesada S, Palacios-Ceña D, et al. (2011) Referred pain from myofascial trigger points in head and neck-shoulder muscles reproduces head pain features in children with chronic tension type headache. $J$ Headache Pain 12: 35-43.

17. Simons DG, Travell J, Simons LS (1999) Myofascial pain and dysfunction: The trigger point manual: Volume 1. $\left(2^{\text {nd }}\right.$ edn), Williams \& Wilkins, Baltimore.

18. Chesterton LS, Sim J, Wright CC, Foster NE (2007) Interrater reliability of algometry in measuring pressure pain thresholds in healthy humans, using multiple raters. Clin $\mathrm{J}$ Pain 23: 760-766.

19. Soee A-BL, Thomsen LL, Tornoe B, Skov L (2013) Reliability of four experimental mechanical pain tests in children. J Pain Res 6: 103-110.

20. Hershey AD, Powers SW, Nelson TD, Kabbouche MA, Winner $P$, et al. (2009) Obesity in the pediatric headache 
population: A multicenter study. Headache 49: 170-177.

21. Ravid S, Shahar E, Schiff A, Gordon S (2013) Obesity in children with headaches: Association with headache type, frequency, and disability. Headache 53: 954-961.

22. Fernández-de-las-Peñas $C$, Alonso-Blanco $C$, Cuadrado ML, Gerwin RD, Pareja JA (2006) Myofascial trigger points and their relationship to headache clinical parameters in chronic tension-type headache. Headache 46: 1264-1272.
23. Von Stülpnagel C, Reilich $P$, Straube A, Schäfer J, Blaschek A, et al. (2009) Myofascial trigger points in children with tension-type headache: A new diagnostic and therapeutic option. J Child Neurol 24: 406-409.

24. Malo-Urriés M, Estébanez-de-Miguel E, Bueno-Gracia E, Tricá-Moreno JM, Santos-Lasaosa S, et al. (2020) Sensory function in headache: A comparative study among patients with cluster headache, migraine, tension-type headache, and asymptomatic subjects. Neurol Sci 41: 2801-2810. 